\title{
Perianales Hidradenoma papilliferum: Diagnose und Differenzialdiagnose
}

\section{Perianal Hidradenoma Papilliferum: Diagnosis and Differential Diagnosis}

Autoren

Institut

\section{S. Boms, A. Enk, W. Hartschuh}

Universitäts-Hautklinik Heidelberg

\section{Bibliografie}

DOI $10.1055 / \mathrm{s}-2007-995481$

Akt Dermatol 2008; 34 :

16-18 ๑ Georg Thieme

Verlag KG Stuttgart · New York ISSN 0340-2541

Korrespondenzadresse Dr. med. Stefanie Boms Universitäts-Hautklinik Ruprecht-Karls-Universität Heidelberg

Vossstraße 2

69115 Heidelberg

Stefanie.Boms@med.uniheidelberg.de

\section{Zusammenfassung \\ $\nabla$}

Das Hidradenoma papilliferum (HP) ist ein seltener, gutartiger Adnextumor mit apokriner Differenzierung, der bevorzugt in der weiblichen Genitalregion auftritt. Gelegentlich wird der Tumor in extragenitaler Lokalisation gefunden. Therapeutisch ist die Exzision mit geringem Sicher-

\section{Einleitung}

Das Hidradenoma papilliferum (HP) ist ein seltener, gutartiger Tumor, der bevorzugt in der weiblichen Genitalregion auftritt. Der Altersgipfel liegt im 3. bis 5. Lebensjahrzehnt. Aufgrund seiner uncharakteristischen Klinik wird das HP häufig verkannt und erst die histologische Aufarbeitung erbringt die Diagnose. Es handelt sich um einen dermal gelegenen Tumor mit apokriner Differenzierung ohne Epidermisbezug.

Gelegentlich wird der Tumor in extragenitaler Lokalisation gefunden. Wir berichten über eine Patientin mit perianalem HP und stellen die wichtigsten klinischen und histologischen Differenzialdiagnosen vor.

\section{Fallbericht \\ V}

\section{Anamnese}

Die 53-jährige Patientin stellte sich mit einem seit $1 \mathrm{Jahr}$ bestehenden Tumor der Perianalregion in der proktologischen Sprechstunde vor. Die Veränderung sei langsam größenprodient gewesen, Beschwerden wie Blutung, Juckreiz oder Schmerzen beim Stuhlgang wurden verneint. Außer einer Alopezia areata totalis waren bei der Patientin keine Vorerkrankungen bekannt. heitsabstand ausreichend, eine Entartung wurde in Einzelfällen diskutiert. Wir stellen eine 53-jährige Patientin mit einem seit 1 Jahr langsam größenprogredienten, symptomlosen, prallen Tumor der Perianalregion vor und diskutieren die Differenzialdiagnosen des Hidradenoma papilliferum in der Perianalregion.

\section{Hautbefund}

In Steinschnittlage zeigte sich bei 7 Uhr perianal ein solitärer, $1,2 \mathrm{~cm} \times 1,0 \mathrm{~cm}$ durchmessender, nicht druckdolenter, halbkugeliger Tumor ohne epidermale Beteiligung ( $\bullet$ Abb.1). Der Tumor war von leicht gelblichem Kolorit, prallelastisch und erschien zystisch aufgebaut.

\section{Histologie}

Die histologische Untersuchung des Exzidates im HE-Schnitt zeigte unter einer vorgewölbten, sonst unauffälligen Epidermis einen vertikal gestellten Knoten mit mehreren zystischen, eosinophiles Sekret enthaltenden Hohlräumen (๑ Abb. 2 a). Daneben fanden sich tubuläre Anteile, die säulenartig in die Hohlräume ragten und von Zylinderepithel ausgekleidet waren. Streckenweise fand sich eine deutliche apokrine Sekretabschnürung (sog. Dekapitationssekretion) ( Abb. 2b).

\section{Therapie}

Nach Exzision des gesamten Tumors in Lokalanästhesie mit einem Sicherheitsabstand von $2 \mathrm{~mm}$ zeigte sich unter lokal antiseptischen Maßnahmen innerhalb von zehn Tagen eine komplikationslose sekundäre Wundheilung. 


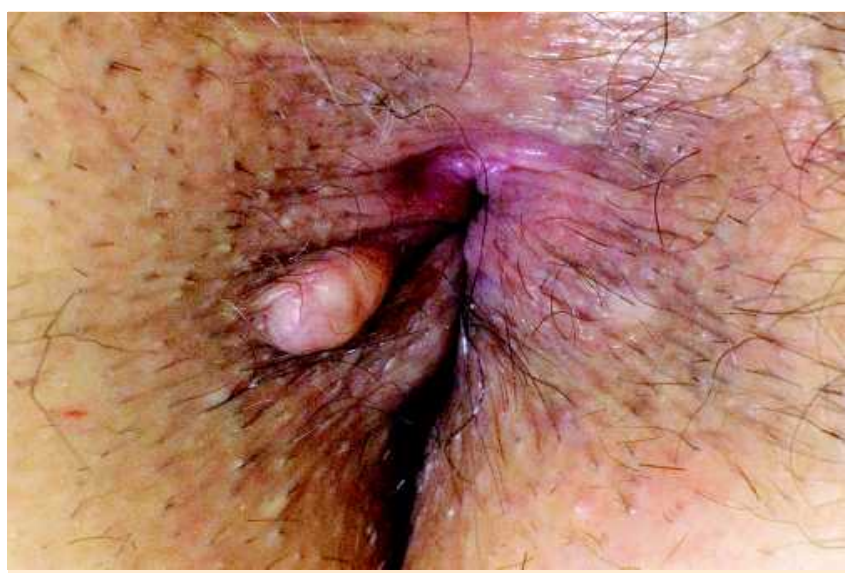

Abb. 1 Solitärer, prallelastischer, indolenter Tumor perianal bei 7 Uhr in Steinschnittlage.
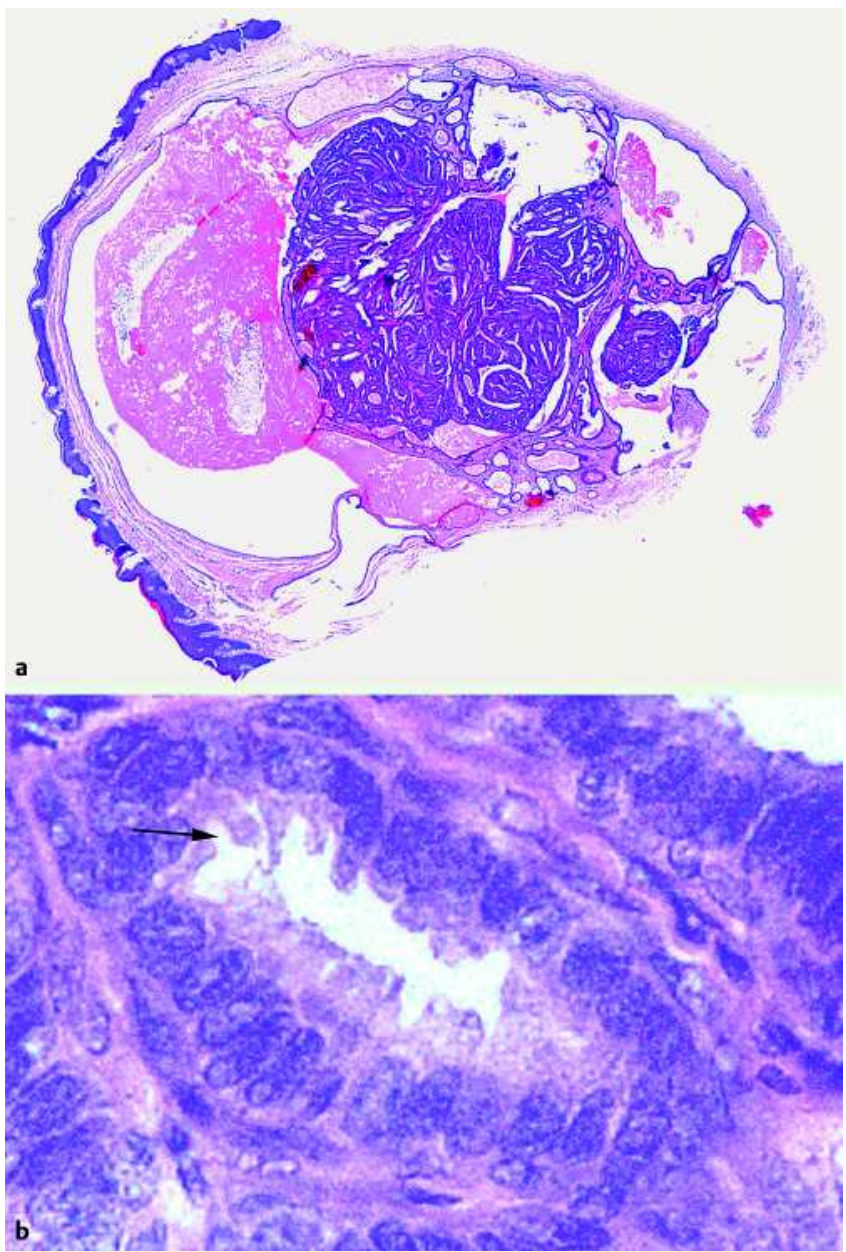

Abb. 2 a Tumor ohne Bezug zur Epidermis, bestehend aus zystischen Hohlräumen ausgekleidet mit Zylinderepithel. HE-Färbung (6-fache Vergrößerung). b Apokrine Sekretabschnürung (Markierung). HE-Färbung (25-fache Vergrößerung).

\section{Diskussion}

$\nabla$

Das Hidradenoma papilliferum (HP) stellt einen benignen Adnextumor mit apokriner Differenzierung dar und gehört in eine heterogene Gruppe seltener benigner und maligner Krankheitsbilder, die bei eher monomorphem und klinisch uncharakteristischem Bild eine außerordentlich große histologische Variabilität aufweisen ( Tab. 1). Der Tumor tritt überwiegend im 3. bis 5 . Lebensjahrzehnt in der weiblichen Genitalregion auf. Die perianale Lokalisation wie im vorliegenden Fall ist 4-fach seltener als die in der Genitalregion, andere Areale wie der Kopfbereich oder das Auftreten bei Männern sind eine Rarität [1 -3]. Klinisch handelt es sich um einen meist solitären, langsam wachsenden, prallelastischen hautfarbenen bis rötlichen Knoten von 1,0 bis $1,5 \mathrm{~cm}$ Größe ohne epidermale Beteiligung. Eine Ulzeration wurde in Einzelfällen beschrieben, ist aufgrund der perianalen Lokalisation gegebenenfalls auch durch Defäkation und Reibung der Nates traumatisch bedingt $[1,4]$.

Tab. 1 Einteilung apokriner Adnextumoren (primär anhand histologischer Kriterien)

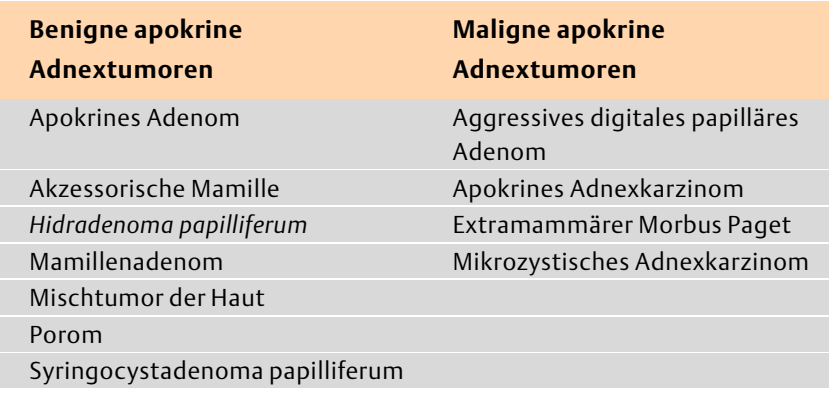

Die Ätiologie des HP ist unklar. Kazakov et al. [3] beschrieben 18 Tumoren mit einem besonderen histologischen Muster, der sogenannten oxyphilen Metaplasie mit eosinophilem Zytoplasma. Des Weiteren wiesen diese Tumoren ein immunhistochemisches Profil ähnlich dem metaplastischen Drüsenepithel der Mamma auf. Es fanden sich milde Kern- und Zytoplasmapleomorphismen. Dadurch kann leicht die Fehldiagnose einer malignen Entartung des HP gestellt werden. Trotz Vorliegen dieser Charakteristika ist der klinische Verlauf des HP auch in diesen Fällen gutartig. Es gibt nur wenige Fallberichte echter maligner Umwandlung. Beschrieben wurden ein intraduktales, kribriformes Karzinom sowie ein adenosquamöses Karzinom auf dem Boden eines HP [5,6]. Außerdem wurde ein koinzidentes Auftreten eines HP und eines extramammären Morbus Paget dokumentiert [7].

Kazakov et al. [3] konnten bei 7/31 untersuchten HP (sowohl gewöhnlicher als auch metaplastischer Tumoren) humane Papillomviren (HPV) der Typen 16, 31, 33, 53 und 56 nachweisen. Die exakte Rolle der HPV ist bislang nicht geklärt.

Aufgrund des klinisch indifferenten Bildes und der Seltenheit des Tumors wird die Diagnose häufig erst nach Exzision und histologischer Aufarbeitung gestellt. Charakteristisch ist die dermale oder subkutane Lage eines zystischen, gekammerten Tumors, der keinen Bezug zur Epidermis hat und aus bizarr verzweigten Epithelzapfen besteht. Diese enthalten neben kuboiden Zellen wenig Tumorstroma und kleiden die Tumorhohlräume mit einem einreihigen Zylinderepithel aus, welches ein eosinophiles 
Tab. 2 Klinische und histologische Differenzialdiagnosen des HP in der Genitoanalregion

\begin{tabular}{ll}
$\begin{array}{l}\text { Klinische Differenzial- } \\
\text { diagnosen }\end{array}$ & $\begin{array}{l}\text { Histologische Differenzial- } \\
\text { diagnosen } \\
\text { Epidermoidzysten }\end{array}$ \\
\hline $\begin{array}{l}\text { Noduläres Basalzellkarzinom } \\
\text { Bartholini Drüsenabszess }\end{array}$ & $\begin{array}{l}\text { Tubuläres apokrines Adenom } \\
\text { Klarzell-Hidradenom }\end{array}$ \\
\hline $\begin{array}{l}\text { Analpolyp } \\
\text { Hämorrhoide }\end{array}$ \\
\hline Perianalthrombose & \\
\hline Mariske &
\end{tabular}

Sekret mittels apokriner Sekretion (sogenannte Dekapitationssekretion) abgibt.

Die klinischen und histologischen Differenzialdiagnosen des HP der Genitoanalregion sind in $\odot$ Tab. 2 dargestellt, wir möchten nur auf die selteneren Diagnosen kurz eingehen.

Das Basalzellkarzinom stellt in der Genitoanalregion eine Rarität dar. In einer histopathologischen Studie an 18943 Basalzellkarzinomen befanden sich $84(0,44 \%)$ in dieser Region, wobei $66 \%$ vom knotigen oder zystischen Typ waren [8]. Damit stellt das ebenfalls solitär und langsam wachsende, indolente Basalzellkarzinom eine wichtige klinische Differenzialdiagnose dar. Die korrekte histologische Diagnose und mikrografisch kontrollierte Exzision ist bei diesem Tumortyp aufgrund der Rezidivfreudigkeit sehr wichtig. Beim HP dagegen ist die einfache Exzision im Gesunden ausreichend.

Das Syringocystadenoma papilliferum stellt einen weiteren Adnextumor mit apokriner Differenzierung und eine wichtige histologische Differenzialdiagnose dar. Es handelt sich um solitäre, manchmal linear auftretende rotbraune Tumoren. Im Gegensatz zum HP treten sie jedoch bevorzugt in der Kopf-Hals-Region auf, oft auf dem Boden eines Naevus sebaceus, und stellen genitoanal eine Rarität dar. Histologisch zeigen sich bei Syringocystadenoma papilliferum mäßig gut abgegrenzte, zottenartige papilläre Tumorkonvolute in der Dermis mit Dekapitationssekretion. Diese gehen jedoch im Unterschied zum HP von der Epidermis aus und erklären die verruköse oder auch ulzerierte Tumoroberfläche. Ein gemeinsames Auftreten eines HP mit Merkmalen des Syringocystadenoma papilliferum wurde kürzlich bei einem Tumor der Vulva beschrieben [4].

\section{Zusammenfassung $\nabla$}

Bei unklaren solitären Tumoren der Genitoanalregion bei Frauen ist es wichtig, das Hidradenoma papilliferum in die klinische Differenzialdiagnose einzubeziehen. In seltenen Fällen muss auch in extragenitaler Lokalisation mit dessen Auftreten gerechnet werden. Die korrekte klinische und histologische Einordnung ist zur Vermeidung weitläufiger Exzisionen infolge der eventuellen Fehldiagnose eines Malignoms unabdingbar.

\section{Abstract}

\section{Perianal Hidradenoma Papilliferum: Diagnosis and Differential Diagnosis $\nabla$}

Hidradenoma papilliferum (HP) is a rare, benign adnexal tumor differentiating towards apocrine glands that predominantly occurs in the female genital region. Occasionally the tumor is located elsewhere. Local excision with a narrow margin is the therapy of choice as malignant transformation is extremely rare. We report a 53-year-old woman with a slowly enlarging symptom free firm perianal tumor and discuss differential diagnosis of hidradenoma papilliferum in the perianal region.

\section{Literatur}

1 Handa Y, Yamanaka N, Inagaki H, Tomita Y. Large ulcerated perianal hidradenoma papilliferum in a young female. Dermatol Surg 2003; 29: $790-792$

2 Minami S, Sadanobu N, Ito T, Natsuaki M, Yamanishi K. Non-anogenital (ectopic) hidradenoma papilliferum with sebaceous differentiation: A case report and review of reported cases. J Dermatol 2006; 33: 256 259

3 Kazakov DV, Mikyskova I, Kutzner H, Simpson RH, Hes O, Mukensnabl P, Bouda J, Zamecnik M, Kinkor Z, Michal M. Hidradenoma papilliferum with oxyphilic metaplasia: a clinicopathological study of 18 cases, including detection of human papillomavirus. Am J Dermatopathol. 2005; 27: $102-110$

4 Nishie W, Sawamura D, Mayuzumi M, Takahashi S, Shimizu H. Hidradenoma papilliferum with mixed histopathologic features of syringocystadenoma papilliferum and anogenital mammary-like glands. J Cutan Pathol. 2004; 31: 561 - 564

5 Pelosi G, Martignoni G, Bonetti F. Intraductal carcinoma of mammarytype apocrine epithelium arising within a papillary hidradenoma of the vulva. Report of a case and review of literature. Arch Pathol Lab Med 1991; 115: 1249-1254

6 Bannatyne P, Elliott P, Russel P. Vulvar adenosquamous carcinoma arising in a hidradenoma papilliferum, with rapidly fatal outcome: case report. Gynecol Oncol 1989; 35: 395-398

7 Stefanato C, Finn R, Bhawan J. Extramammary paget disease with underlying hidradenoma papilliferum. Am J Dermatopathol 2000; 22: 439-442

8 Gibson GE, Ahmed I. Perianal and genital basal cell carcinoma: A clinicopathologic review of 51 cases. J Am Acad Dermatol 2001; 45: 68 - 71 